\title{
Light-scattering gating and characterization of plasma microparticles
}

\author{
Anastasiya I. Konokhova \\ Darya N. Chernova \\ Dmitry I. Strokotov \\ Andrei A. Karpenko \\ Andrei V. Chernyshev \\ Valeri P. Maltsev \\ Maxim A. Yurkin
}




\title{
Light-scattering gating and characterization of plasma microparticles
}

\author{
Anastasiya I. Konokhova, ${ }^{a}$ Darya N. Chernova, ${ }^{a, b}$ Dmitry I. Strokotov, ${ }^{a, c}$ Andrei A. Karpenko, ${ }^{d}$ \\ Andrei V. Chernyshev, ${ }^{a, b}$ Valeri P. Maltsev ${ }^{a, b, c}$ and Maxim A. Yurkin ${ }^{a, b, *}$ \\ ${ }^{a}$ Voevodsky Institute of Chemical Kinetics and Combustion SB RAS, Institutskaya 3, 630090 Novosibirsk, Russia

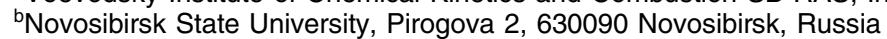 \\ "Novosibirsk State Medical University, Krasny Prospect 52, 630091 Novosibirsk, Russia \\ dState Research Institute of Circulation Pathology, Rechkunovskaya 15, 630055 Novosibirsk, Russia
}

\begin{abstract}
Flow cytometry method (FCM) is widely used for analysis of cell-derived microparticles (MPs). Numerous efforts are currently aimed to standardize these measurements among different instruments. We push the FCM characterization of MPs to the limit based on rigorous simulation of measured signals. We measured forward- and side-scatter (FSC/SSC) signals and angle-resolved light-scattering profiles (LSPS) of polystyrene microspheres and MPs, including their aggregates, using a scanning flow cytometer (SFC). We used the Mie theory to (1) accurately evaluate instrument detection limits; (2) construct FSC/SSC gates for MPs in absolute scales of size and refractive index (RI); and (3) determine size and RI of individual spherical MPs. LSPs were used for advanced characterization, including differentiation of spherical and nonspherical particles. The proposed absolute FSC/SSC gating is naturally standardized for any FCM instrument, given the knowledge of its optical system and leads to instrument-independent analysis of MPs. The inverse Mie problem has a unique solution only for some regions of size and RI and uncertainties rapidly increase with decreasing size and RI. The developed methods are applicable to any flow cytometer, but are limited by assumption of particle sphericity. The latter can be relaxed only if additional signals, such as LSP, are measured. ๑ 2016 Society of Photo-Optical Instrumentation Engineers (SPIE) [DOI: 10.1117/1.JBO.21.11.115003]
\end{abstract}

Keywords: Cell-derived microparticles; flow cytometry; light scattering; limit of detection; standardization; inverse problem. Paper 160591R received Aug. 28, 2016; accepted for publication Oct. 27, 2016; published online Nov. 17, 2016.

\section{Introduction}

Cell-derived microparticles (MPs) are plasma membrane structures, which are released directly from cell surfaces into the extracellular space under both physiological and stress conditions and normally circulate in peripheral blood and body fluids. ${ }^{1,2}$ Along with exosomes, MPs represent two types of extracellular vesicles, which attract constantly growing research interest as an important mode of intercellular communication relevant in health and disease and as potential clinical diagnostic and therapeutic targets. ${ }^{3-5}$ However, being formed by different mechanisms, MPs constitute a heterogeneous population highly variable by size, composition, density, and cell of origin. For several decades, the flow cytometry method (FCM) has been the gold-standard method for measuring cell-derived MPs, suitable for routine studies in clinical laboratories and for multiparametric single-particle measurements. ${ }^{6,7}$ The standard FCM forward (FSC) and side scatter (SSC) measurements, as well as fluorescent measurements based on labeling techniques, are commonly used to enumerate, classify, and size various biological objects in suspensions. Small MP sizes challenge sensitivity and resolution of FCM instruments; still they have long been used in an uncritical manner. ${ }^{8}$ Only recently has it been generally appreciated that reliability and efficiency of FCM detection of MPs significantly depend on a variety factors, including optical configuration of the particular instrument, mode of triggering (SSC-, FSC-, or fluorescence-based), and the threshold

*Address all correspondence to: Maxim A. Yurkin, E-mail: yurkin@gmail.com level, along with appropriate use of reference particles and adequate calibration protocols. ${ }^{9,10}$ Numerous efforts are currently aimed at standardizing these procedures to make FCM measurements of MPs comparable between different instruments. Recent studies ${ }^{11}$ have addressed some of the shortcomings and provided a deeper insight, but much work remains.

On the one hand, the potential of the FCM is still underused for MPs. For instance, absolute determination of size and refractive index (RI) for homogeneous spherical particles based on simultaneous FSC and SSC measurements was proposed 20 years ago. ${ }^{12-14}$ Although this approach has its limitations, even it has not been yet applied to MP characterization. The Mie theory was firmly established as a rigorous simulation tool a few years ago, ${ }^{15}$ but the currently confirmed capabilities are mainly limited to adequate size-related gatings based on either FSC or SSC. ${ }^{16,17}$ Still it strongly depends on the variability of MPs RI, which has to be fixed a priori. ${ }^{18}$

On the other hand, MPs are definitely not an easy object for the FCM resulting in inherent limitations. While several alternative methods for single-particle MP characterization exist: nanoparticle tracking analysis (NTA), ${ }^{19,20}$ resistive pulse sizing (RPS), ${ }^{21,22}$ atomic-force microscopy, ${ }^{23}$ and cryo-electron microscopy, ${ }^{24,25}$ most of them cannot claim to be superior to the FCM due to various complications. ${ }^{7,26}$ There is, however, a promising technology-scanning flow cytometry (SFC), ${ }^{27,28}$ measuring angle-resolved light-scattering profiles (LSPs) of single particles in a flow. In application to MPs, it provides 
not only calibration-free robust determination of the size and RI of each particle ${ }^{29}$ but also a certain level of shape awareness, including differentiation of spherical and nonspherical MPs. ${ }^{30}$ The shape uncertainty is a major issue for MP that may cause problems for most of the other methods, including the FCM, NTA, and RPS. ${ }^{18}$

The main goal of this methodological paper is to push the flow-cytometry technology (both FCM and SFC) to the limit specifically for MP characterization and to provide guidelines for further hardware development and standardization. The key discussed questions are (1) assessment of the instrument sensitivity for MP detection based on standard FSC and/or SSC measurements; (2) which MP's characteristics can be estimated from these scatter measurements; (3) additional capabilities provided by angle-resolved light-scattering measurements. We propose an approach to standardize scatter-based detection of MPs, taking into account the FCM optical configuration. This includes accurate evaluation of the instrument sensitivity and instrument-independent gating strategy in absolute scales of size and RI. Moreover, we propose a potential method to determine size and RI of single-spherical MPs from the FSC and SSC signals and analyze its feasibility in terms of uniqueness and accuracy of the solution. This leads to complete understanding of the instrument capabilities in MP detection and characterization. Finally, we contrast those best-of-the-FCM-world results with the capabilities of the SFC, including shape classification and uncertainties of estimated MP characteristics.

\section{Materials and Methods}

\subsection{Scanning Flow Cytometer}

An SFC (Cytonova LLC Company, Novosibirsk, Russia) was used to measure standard FSC and SSC, as well as angular dependence of the intensity of the scattered light in a wide range of polar angles, or angle-resolved LSPs, of individual particles. ${ }^{31}$ Technical features of the SFC were described in detail elsewhere. ${ }^{27,28}$ LSPs of individual particles in flow were generated with a $405 \mathrm{~nm}$ laser (30 mW, Radius, Coherent Inc., Santa Clara, USA), and FSC and SSC were measured at $488 \mathrm{~nm}$ (15 mW laser, FCD488-020, JDS Uniphase Corporation, Milpitas). By considering only the latter signals, we emulate a representative example of a standard flow cytometer. Note, however, that the FSC/SSC collection angles vary a great deal among existing instruments, so the specific gating results of this paper should be generalized with caution. In particular, SFC has the FSC detector integrating over a polar angle of 14.2 to $23 \mathrm{deg}$ and the SSC detector with an NA of 0.5 . The corresponding collection angular ranges both for FSC and SSC were estimated from analysis of a mixture of different PS microspheres, similar to that proposed by Fattaccioli et al., ${ }^{32}$ and summarized in Table 1.

Within the Mueller-matrix formalism ${ }^{33}$ for description of light scattering, the LSP, FSC, and SSC intensities (FSC/SSC peak amplitudes) measured by the SFC are expressed as

$$
\begin{aligned}
I_{\mathrm{LSP}}(\theta)= & k_{\mathrm{LSP}} \int_{0}^{2 \pi} \mathrm{d} \varphi\left[S_{11}(\theta, \varphi)+S_{14}(\theta, \varphi)\right], \\
I_{\mathrm{SSC} / \mathrm{FSC}}= & k_{\mathrm{SSC} / \mathrm{FSC}} \iint_{\Omega_{\mathrm{SSC} / \mathrm{FSC}} \mathrm{d} \theta \mathrm{d} \varphi \sin \theta\left[S_{11}(\theta, \varphi)\right.} \\
& \left.-S_{12}(\theta, \varphi) \cos (2 \varphi)-S_{13}(\theta, \varphi) \sin (2 \varphi)\right],
\end{aligned}
$$

where $S_{i j}(\theta, \varphi)$ are elements of the Mueller matrix, $\theta$ and $\varphi$ are

\begin{tabular}{|c|c|c|}
\hline & Spherical MPs/PS microspheres & MPs dimers/doublets of PS microspheres \\
\hline \multirow{5}{*}{$\begin{array}{l}\text { Optical model and particle } \\
\text { characteristics }^{30}\end{array}$} & Sphere & Bisphere \\
\hline & & Diameter $d_{1}$ \\
\hline & Diameter $d$ & Diameter $d_{2}$ \\
\hline & $\mathrm{RI} n$ & Effective RI $n$ \\
\hline & & Polar orientation angle $\beta$ \\
\hline Simulation method & The Mie theory ${ }^{33}$ & $\begin{array}{l}\text { The discrete-dipole approximation }{ }^{34} \\
\text { (code ADDA } 1.2^{35} \text { ) }\end{array}$ \\
\hline Laser wavelength (LSP) & $405 \mathrm{~nm}$ & \\
\hline Laser wavelength (FSC/SSC) & $488 \mathrm{~nm}$ & \\
\hline LSP angular range & [15 deg, $50 \mathrm{deg}]$ & \\
\hline FSC collection angles & [14.2 deg, $23 \mathrm{deg}$ ] (annular aperture) & \\
\hline SSC collection angles & $90 \operatorname{deg} \pm 18.3 \mathrm{deg}$ (circular aperture) & \\
\hline \multirow[t]{2}{*}{ Medium RI $(0.9 \% \text { saline })^{36}$} & 1.345 (at wavelength $405 \mathrm{~nm}$ ) & \\
\hline & 1.339 (at wavelength $488 \mathrm{~nm}$ ) & \\
\hline
\end{tabular}
the polar and azimuthal scattering angles, $k_{\mathrm{LSP}}$ and $k_{\mathrm{FSC} / \mathrm{SSC}}$ are

Table 1 Optical models, methods, and system parameters, used for light-scattering simulations of reference microspheres and plasma MPs.

Note: MPs, microparticles; PS, polystyrene; RI, refractive index; LSP, light-scattering profile; FSC, forward scatter; SSC, side scatter. 
the scaling coefficients, and $\Omega_{\mathrm{FSC} / \mathrm{SSC}}$ are the solid angles, determined by SFC detection optics. All experimental and theoretical LSPs presented in this study are additionally multiplied by the weighting function

$w(\theta)=\frac{1 \mathrm{deg}}{\theta} \exp \left\{-2 \log ^{2}[\theta /(54 \mathrm{deg})]\right\}$,

which is an approximation of the SFC transfer function and provides an uniform experimental-noise level over the considered angular region. ${ }^{37}$

The operational angular range in which SFC measures angleresolved light-scattering information, or LSP, was determined from analysis of $1.87 \mu \mathrm{m}$ PS microspheres, as described elsewhere, ${ }^{37}$ to be from 15 to $50 \mathrm{deg}$ with a resolution of $0.5 \mathrm{deg}$ (Table 1). FSC and SSC threshold levels were set to two standard deviations of the noise in corresponding channels. FSC provided a wider detection range for single-spherical particles (see Fig. 1), and, therefore, was used to trigger the electronics of the SFC. Moreover, we discarded particles with $S / N$ for the LSP signal $<1$ to avoid accidental detection of background noise. Since the LSP is originally a time-resolved signal measured over $600 \mu \mathrm{s},{ }^{28}$ as compared to $<10 \mu \mathrm{s}$ for FSC/SSC signals, it allows us to reliably detect coincident events (so-called, swarm detection) and to remove them from further consideration.

\subsection{Plasma Microparticles and Reference Particles}

To eliminate the impact of preanalytical steps, including loss of MPs during centrifugation, and to preserve natural MP characteristics, we performed MP measurements directly in fresh platelet-rich plasma as described previously. ${ }^{30}$ The whole blood sample was obtained after informed consent from a healthy volunteer and collected in a vacuum citrate tube (BD Vacutainer Systems, BD, UK). A supernatant of platelet-rich plasma formed in a tube within $2 \mathrm{~h}$ of the collection by natural precipitation was carefully collected and diluted by 30 -fold in $0.2 \mu \mathrm{m}$ filtered (Sartorius Stedim Biotech GmbH, Goettingen, Germany)
$0.9 \%$ saline containing preliminarily added 0.7 and $1.87 \mu \mathrm{m}$ reference PS microspheres. While platelets are removed from consideration after LSP processing based on their size and shape, ${ }^{30}$ the remaining events may comprise plasma components other than MPs, such as lipoprotein particles (LPs), including chylomicrons, the largest and least dense lipoprotein species. ${ }^{38}$

We used nonfluorescent polystyrene (PS) microspheres, including Molecular Probes 0.4, 0.7, 1, and $3 \mu \mathrm{m}$, and Thermo Scientific $1.87 \mu \mathrm{m}$, to determine LSP, FSC, and SSC light acceptance angular ranges, as well as in actual measurements together with MPs. As optical models for both reference beads and MPs, we considered spheres and bispheres-their characteristics and corresponding light-scattering simulation methods are summarized in Table 1.

\subsection{FSC/SSC-Based Particle Characterization}

Once intrinsic technical characteristics of FCM are determined, the scatter signals for a homogeneous spherical particle depend only on diameter and RI. Therefore, particle characterization, the so-called inverse Mie problem, boils down to solving the following system of two equations

$I_{\mathrm{FSC}}^{\mathrm{exp}}=I_{\mathrm{FSC}}^{\mathrm{th}}(d, n)$,

$I_{\mathrm{SSC}}^{\mathrm{exp}}=I_{\mathrm{FSC}}^{\mathrm{th}}(d, n)$,

where superscripts "exp" and "th" denote experiment and theory, respectively. However, neither existence nor the uniqueness of the solution is guaranteed a priori. While we have not encountered any practical problems with the existence, the multiple solutions do exist for many values of size and RI [see Fig. 3(a)]. Thus, we solved the system using the LevenbergMarquardt method in a multistart mode, which reliably finds all existent solutions. We also estimated the uncertainties of the obtained characteristics using the sampling method, varying the FSC and SSC signals within the experimental errors.
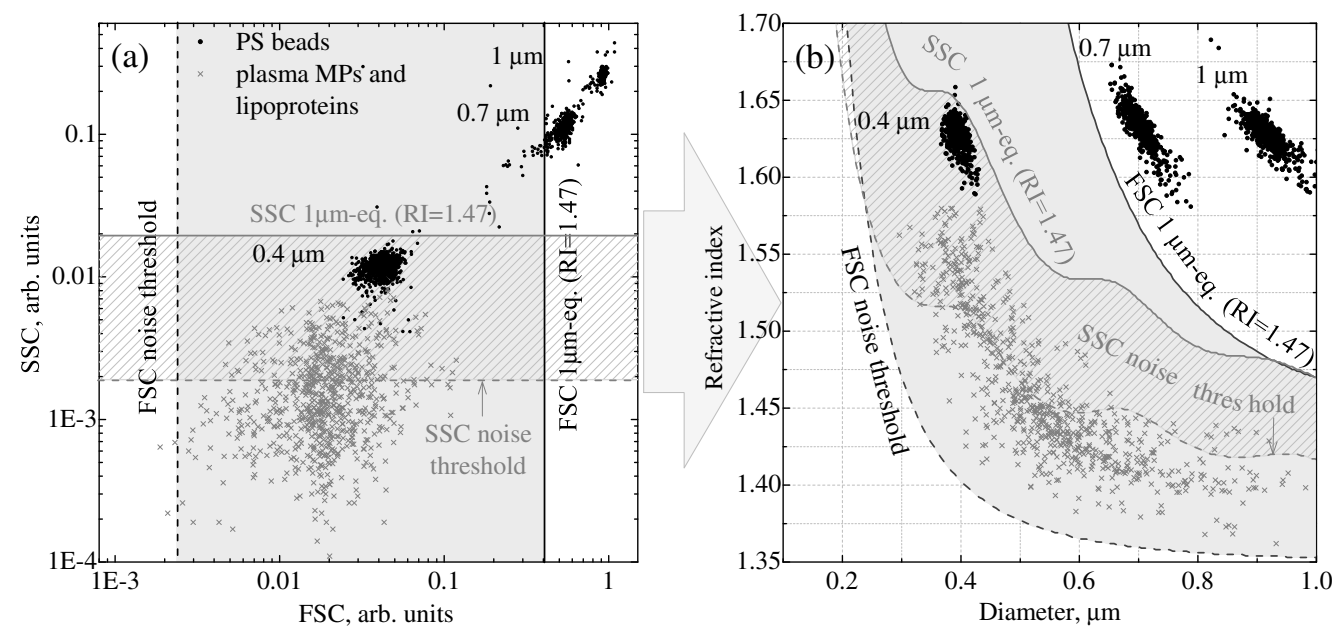

Fig. 1 (a) Standard MP gating based on FSC and SSC levels equal to that of an equivalent spheregray-colored and hatched regions, respectively. Dashed lines are the FSC (black) and SSC (gray) detection limits, solid lines are upper limits, corresponding to the signal of $1-\mu \mathrm{m}$ particle with RI of 1.47. The experimental data to illustrate the gating are for three reference PS beads $(0.4,0.7$, and $1 \mu \mathrm{m})$ and for a spherical fraction of plasma particles, identified using their LSPs. (b) The same gates, but in coordinates of RI versus diameter, computed using the Mie theory. Corresponding particle characteristics are obtained through the solution of the ILS problem. 


\subsection{Particle Characterization From Angle-Resolved Scatter Measurements}

A more advanced approach is based on simultaneous fitting of experimental LSPs, FSC, and SSC signals by theoretical ones. Unfortunately, the FSC and SSC amplitudes can be used only for spherical particles, because otherwise they depend on the azimuthal orientation angle (in addition to the polar orientation angle $\beta$ ), in contrast to the LSP, which is integrated over the azimuthal scattering angle [see Eq. (1)]. The inverse light-scattering (ILS) problem was transformed into the global minimization of the following weighted sum of residual squares:

$$
\begin{aligned}
S(\mathbf{Q})= & \sum_{i=1}^{N}\left[w\left(\theta_{i}\right)\right]^{2}\left[\frac{I_{\mathrm{LSP}}^{\exp }\left(\theta_{i}\right)-\alpha I_{\mathrm{LSP}}^{\mathrm{th}}\left(\theta_{i}, \mathbf{Q}\right)}{\sigma_{\mathrm{LSP}}}\right]^{2} \\
& +\left[\frac{I_{\mathrm{SSC}}^{\exp }-I_{\mathrm{SSC}}^{\mathrm{th}}(\mathbf{Q})}{\sigma_{\mathrm{SSC}}}\right]+\left[\frac{I_{\mathrm{FSC}}^{\mathrm{exp}}-I_{\mathrm{FSC}}^{\mathrm{th}}(\mathbf{Q})}{\sigma_{\mathrm{FSC}}}\right]^{2},
\end{aligned}
$$

where $\mathbf{Q}$ is the vector of particle characteristics $(\{d, n\}$ for spheres and $\left\{d_{1}, d_{2}, n, \beta\right\}$ for bispheres), $N$ is a number of LSP points, $\sigma_{\mathrm{LSP} / \mathrm{FSC} / \mathrm{SSC}}$ are the experimental uncertainties of scatter measurements, and $\alpha$ is the coefficient that compensates for the effect of the deviation of particle trajectory from the main axis in the flow cell of the SFC.

Two global optimization algorithms were used to minimize $S(\mathbf{Q})$ : DiRect $^{37,39}$ for spheres and nearest-neighbor interpolation using the precalculated database of LSPs for bispheres. ${ }^{40}$ Both algorithms not only determine the best-fit characteristics or their mathematical expectations, but also estimate their errors based on the Bayesian method. ${ }^{40}$ Moreover, estimated uncertainties adequately respond (by larger errors of characteristics) to model errors, e.g., when a nonspherical particle is fitted by a spherical model. ${ }^{37}$ Applying both shape models to each MP and PS microsphere, we chose the best model based on goodness of LSP fit using the Bayesian information criterion. ${ }^{30}$

Using the signals for two wavelengths is complicated by the dispersion of RI. To keep the number of characteristics low, we assume that RI at $488 \mathrm{~nm}$ is fully determined by its value at $405 \mathrm{~nm}$. In particular, we assume the constant difference for PS microspheres according to the bulk values, ${ }^{41}$ i.e., $n(488 \mathrm{~nm})=$ $n(405 \mathrm{~nm})-0.023$. By contrast, for MP, we assume the RI dispersion to be comparable to that of the medium, i.e., $n(488 \mathrm{~nm})=0.99554 \times n(405 \mathrm{~nm})$, which is equivalent to considering a constant RI relative to the medium. To allow direct intercomparison of our results, we further report only $n(405 \mathrm{~nm})$, even when the originally determined characteristic is $n(488 \mathrm{~nm})$.

\section{Results}

\subsection{FSC/SSC-Based Triggering and Gating}

Consider the gating strategy ${ }^{10,42}$ based on setting the FSC and/or SSC levels equal to that of a sphere with specific size and RI. We illustrate this strategy in Fig. 1(a), assuming $d=1 \mu \mathrm{m}$ and $n=1.47$, together with detection (noise) thresholds. Typically, the values from 1.38 to 1.4 are used as RI of MPs ${ }^{16,17,26}$ with no convincing justification. Although the distribution of MPs over RI were obtained with the NTA, ${ }^{43}$ they are too wide (1.36-1.55) for practical usage. Since this width is due to the uncertainty of single measurements, even mean and mode values of the distributions may be significantly biased. However, there are evidences that in fresh platelet-rich plasma MPs are commonly accompanied by LPs, which have larger RI. ${ }^{24}$ Hence, we used the value of $n=1.47$ as a tentative upper limit for MPs, e.g., to separate them from LPs. ${ }^{44}$

In the FSC/SSC [Fig. 1(a)] map, we show three reference PS microspheres $(0.4,0.7$, and $1 \mu \mathrm{m})$ and all spherical plasma particles, identified and characterized using their LSPs. The measurements for PS beads are adequately positioned with respect to the gates, considering the larger RI of PS. Being mapped to size/ RI coordinates [Fig. 1(b)], these gates represent curved regions that clearly demonstrate the nonlinear (not even monotonous) dependence of the FSC/SSC signals on particle characteristics. For our instrument, the FSC provides a wider (than SSC) detection range with 200-nm PS microspheres and 300-nm MP at its edge. Size/RI representation of the FSC-based gate is elongated along RI and does not allow one to select particles in a specific range, e.g., plasma MPs with $\mathrm{RI}<1.47$ or in a particular size range. Thus, Fig. 1(b) shows serious limitations of the standard gating strategy, but immediately points to evident improvement, which we name "absolute gating." The latter operates directly in size/RI coordinates [Fig. 2(a)] and is further transformed into the instrument-specific scatter gates [Fig. 2(b)]. First, we constructed a regular coordinate grid in size/RI and transformed it into the scatter coordinates, showing the complexity of the underlying Mie mapping. In particular, the mapping is not injective; hence, some of the grid lines intersect. Second, we show three exemplary gates: one for $0.4 \mu \mathrm{m}$ microspheres and two RIbased gates for the plasma constituents. As mentioned above, the RI values of MPs and their relation to that of LPs are known very poorly. We leave a detailed study of this interesting issue for future research; here we specify the RI ranges ([1.37,1.47] and [1.47,1.58] for MPs and LPs, respectively) rather arbitrarily just to illustrate the capabilities of this strategy. Other meaningful gates can be constructed analogously if a more detailed specification of size and RI ranges is available. The LSP-based sizing is robust but it does not exactly conserve FSC and SSC values, which explains why $15 \%$ of plasma components seemingly fall into the wrong FSC/SSC gates.

Finally, the proposed gating strategy does not always require both FSC and SSC signals to be above the noise thresholds. For instance, a single significant signal (FSC) is sufficient to detect a nonnoise event, and the specific SSC value (up to a noise threshold) does not matter as long as it falls inside the gate [as with $\mathrm{G}_{\mathrm{MP}}$ in Fig. 2(b)].

\subsection{Is it Possible to Determine Size and RI from FSC/SSC Measurements?}

The natural next step is to invert the Mie mapping from size/RI to FSC/SSC and use it to determine the former characteristics for each measured particle. We theoretically analyzed the feasibility of such a characterization approach in the range $d \in$ $[0.1,1.5] \mu \mathrm{m}$ and $n \in[1.35,1.7]$, covering both MPs and reference PS microspheres. We sampled this range with a $100 \times 100$ grid and computed the scatter signals for each grid point. Equations (4) and (5) were then solved, and the resulting number of solutions is shown in Fig. 3(a). For the current SFC optical configuration, the solution is unique only for a limited range of characteristics, part of which is below the instrument detection limits. Even if a solution is unique or a proper one can be selected based on a priori information, such as a narrow size or $\mathrm{RI}$ range, its usefulness is determined by estimation uncertainties. The latter are plotted in Figs. 3(b) and 3(c), showing that for 


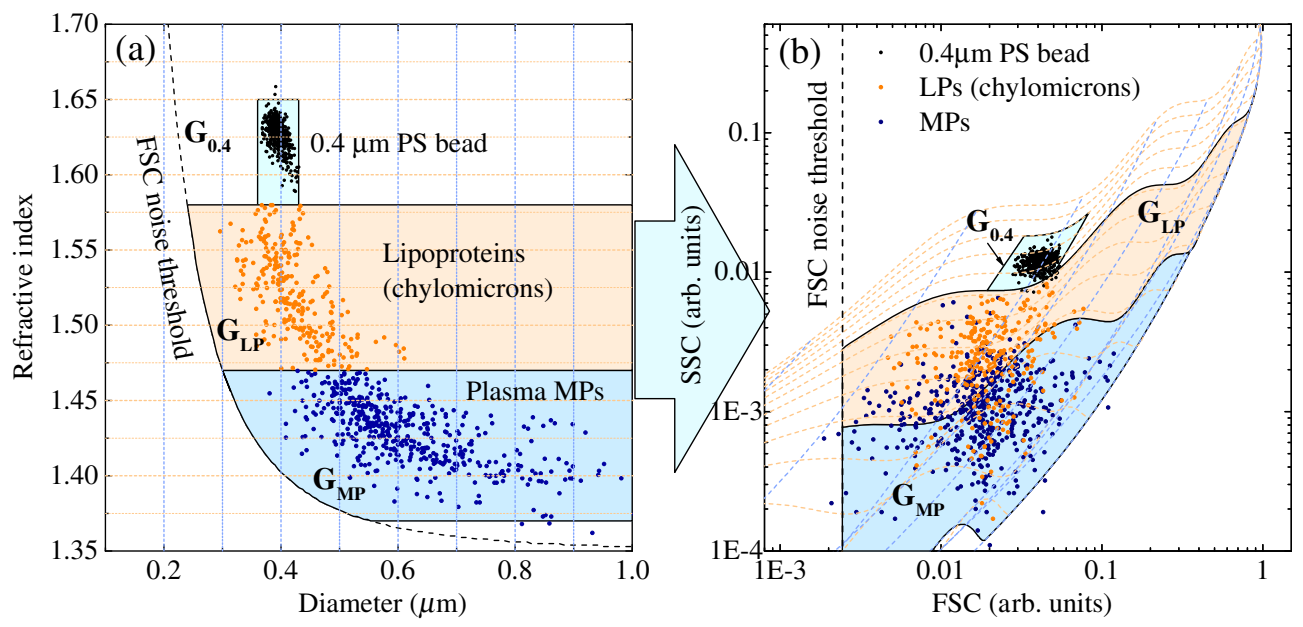

Fig. 2 Absolute MP gating strategy in terms of size/RI. Three gates are defined in (a): $G_{0.4}$ is $[0.36,0.43] \mu \mathrm{m}$ for diameter and $[1.58,1.65]$ for $\mathrm{Rl}$, tentative MP and $L P$ gates $\left(\mathrm{G}_{M P}\right.$ and $\left.\mathrm{G}_{L P}\right)$ are defined by $R I$ ranges [1.37,1.47] and [1.47,1.58], respectively, upper size limit of $1 \mu \mathrm{m}$, and lower size limit along the FSC threshold. (b) The same gates in FSC/SSC coordinates together with grid lines of constant size and $\mathrm{RI}$ (dashed lines). The experimental data to illustrate the gating are for $0.4-\mu \mathrm{m}$ PS beads and for a spherical fraction of plasma particles, including MPs and LPs, processed using their LSPs.

single-spherical particles with $n \geq 1.5$, including silica or PS microspheres, size, and RI could be deduced with relative standard deviation less than $10 \%$. However, in the range of MPs, uncertainties may exceed $30 \%$, mostly due to the $S / N$ ratio decreasing with both size and RI. There is also an inherent limitation related to the Rayleigh scattering regime (particles much smaller than the wavelength), at which all optical properties are determined by particle polarizability. ${ }^{33}$ The latter is a function of size and RI, which cannot be discerned individually from any optical measurements (with a given wavelength). More specifically, the relative sensitivity of inverse mapping (uncertainties for a constant $S / N$ ratio) increases approximately as $d^{-2}$ (considering only the first two terms of the Mie series). Coupled with $d^{6}$ scaling of the scatter signals, it results in very unfavorable scaling of uncertainties as $d^{-8}$ for a constant noise level. While there is no hard boundary, we calculated a tentative one corresponding to $100 \%$ uncertainties of either $d$ or RI for a noise level $10^{4}$ times smaller than the current one. It is indicated by the black color in Figs. 3(a)-3(c).

Finally, Figs. 3(d)-3(g) show typical examples corresponding to different multiplicities of the solution. For instance, the SFC cannot resolve a $0.71-\mu \mathrm{m}$ PS microsphere and a particle of
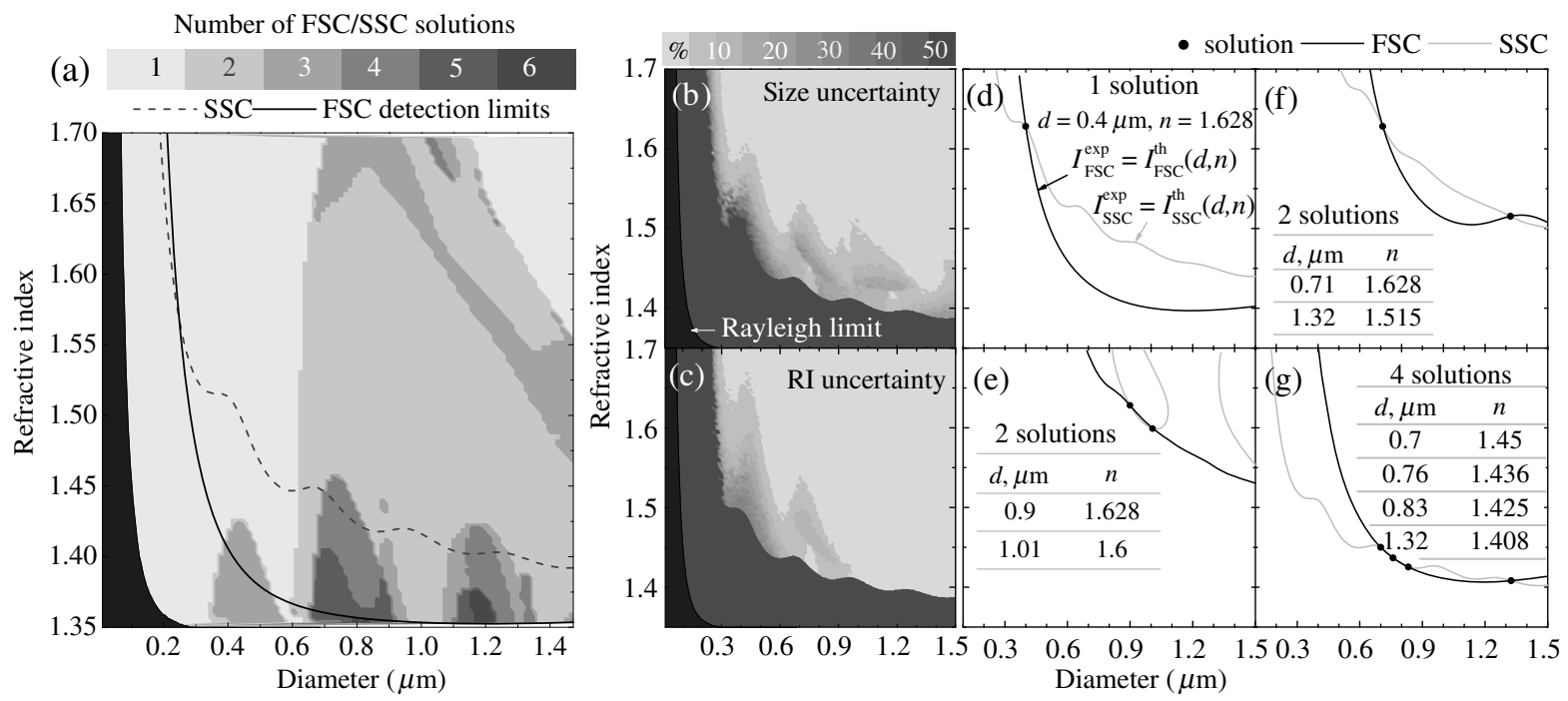

Fig. 3 (a) Contour plot of number of solutions (ambiguity) of inverse Mie problem for a sphere from FSC/ SSC measurements versus its size and RI. Current FSC and SSC detection limits are also shown. (b) and (c) are same as (a), but for relative uncertainty (standard deviations) of size (top) and RI (bottom) estimates, assuming constant noise amplitudes in scatter channels (based on experimental data). (d)-(g) Typical examples illustrating the cases of unique and multiple solutions, including single PS microspheres [ $d=0.4,0.71$, and $0.9 \mu \mathrm{m}$ with $n=1.628-(\mathrm{d})$, (f), and (e) respectively] and a potential MP (g) with $d=0.7 \mu \mathrm{m}$ and $n=1.45$. The solutions are shown as intersections of constant-level curves for FSC and SSC. 

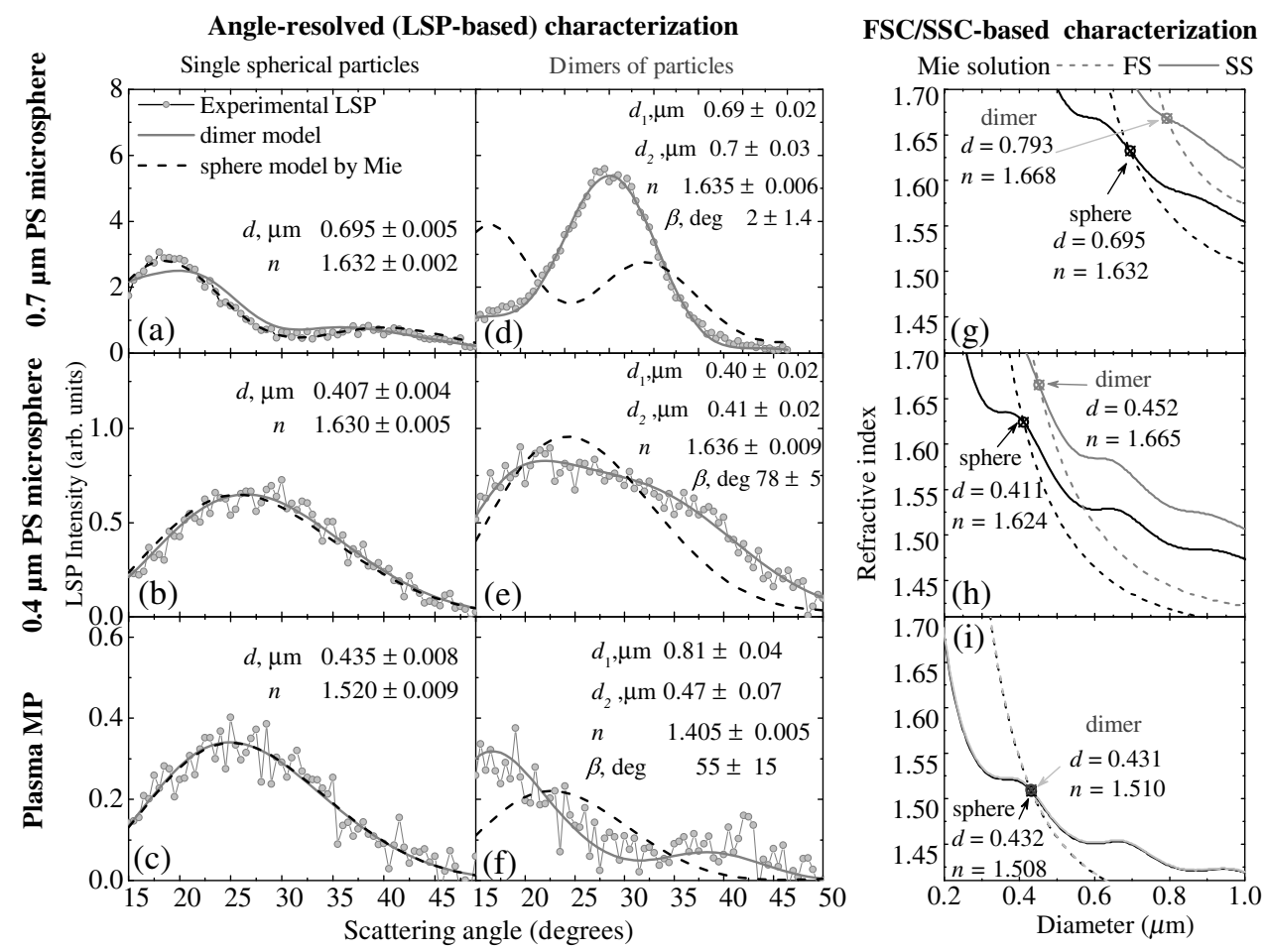

Fig. 4 Application of FSC/SSC-based (g)-(i) and angle-resolved LSP-based (a)-(f) characterization to single-submicron particles, including 0.7 (top) and $0.4 \mu \mathrm{m}$ (middle) PS microspheres, and plasma MPs (bottom). The solutions of corresponding ILS problems are demonstrated for single-spherical particles (a)-(c) and their dimers (d)-(f). Experimental and best-fit theoretical LSPs (for sphere and dimer models), as well as estimates of particle characteristics (mathematical expectation \pm standard deviation) of the best-fit model are also shown. (g)-(i) FSC/SSC-based solutions shown as intersections of constant-level curves of FSC/SSC scatter intensities. In (i), those curves are almost the same for the considered particles.

$d=1.32 \mu \mathrm{m}$ and $n=1.515$ based on FSC/SSC measurements. However, the ambiguity can be removed if one assumes the material to be PS (hence, restricting RI to a narrow range). For a $0.9-\mu \mathrm{m}$ microsphere, a second solution is closer and cannot be easily gated out. The number of solutions generally increases with decreasing RI; an example of a potential MP is given with four solutions, three of them forming a singleconfidence region.

\subsection{Characterization of Single Particles: FSC/SSC versus Angle-Resolved Light Scattering}

The goal of this section is to show the effect of particle shape (nonsphericity) on the characterization results. For that, we applied both FSC/SSC- and LSP-based approaches to characterize measured PS microspheres ( 0.4 and $0.7 \mu \mathrm{m})$ and plasma MPs. MPs are known to be present in plasma not only as spheres, but also as dimers (doublets) of spheres and more complicated shapes. ${ }^{24,25,30}$ Similarly, 5\% of PS microspheres were in a form of dimers, despite sonication. We show in Fig. 4 several illustrative examples from our data that were unambiguously identified as either monomer or dimer of spheres, based on their LSP. For presented single-spherical particles, both FSC and SSC signals were above the noise threshold, and the solution of the corresponding inverse Mie problem is either unique or can be made such by prior RI gating. Moreover, both approaches led to similar results for size and RI within their uncertainties.

By contrast, dimers highlight the difference between the two approaches. LSP measurements allow one to identify and accurately characterize those dimers, while FSC/SSC approach results in a predictable failure. For presented dimers of 0.4- and $0.7-\mu \mathrm{m}$ PS spheres, both the resulting size and RI are wrong. In principle, the presence of dimers of PS beads (or other monodisperse particles) is not an issue, since those dimers can be easily identified and removed from consideration based on their larger FSC and SSC intensities or on resulting characteristics falling outside of the expected range [Figs. 4(g) and 4(h)]. The situation is markedly different for polydisperse samples, such as MPs. We have chosen two events, corresponding to a dimer of MPs and a single denser spherical MP (probably, an LP one), having almost the same FSC and SSC signals [overlapping constant-level FSC/SSC curves in Fig. 4(i)]. Hence, FSC/SSCbased characterization has no capabilities whatsoever to distinguish between those two events.

\section{Discussion}

We proposed a general approach to standardize FCM measurements of MPs based on rigorous simulation of the measured signals. While all specific results (e.g., detection thresholds or number of solutions of the inverse Mie problem) are related to the particular used instrument (SFC), they do illustrate potential capabilities and limitations of the advocated approach when applied to any flow cytometer. Also note that the FSC/SSC collection angles are critical components of the instrument optical model. They can either be obtained from the instrument documentation or determined independently based on measurement of reference particles. ${ }^{10,14,16,32}$ However, the accuracy of 
the provided estimates and ways to improve it are important topics for future research.

The first benefit of an instrument optical model is meaningful scatter gates based on absolute values of size and RI (Fig. 2). This is a natural next step compared to both "relative sizing" (gating based on scatter signals of PS beads) ${ }^{17}$ and single-signal analysis, in which reference beads are used to determine the calibration factors. ${ }^{16}$ The limitations of the previous approaches are mainly related to a complex nonlinear dependence of scatter signals on both particle characteristics and FCM optical configuration, as emphasized in Refs. 30 and 45. These limitations are also evident from Fig. 1(b), while the proposed two-signal approach is a ready-to-use solution to this longstanding problem. We have illustrated the gates with the LSP-processed data, but these data are not used for gates construction. Therefore, this strategy can be applied to any flow cytometer, and any region in size/RI coordinates can be implemented as FSC/SSC gate. The remaining limitation is poor knowledge of the RI of MPs, including other plasma components such as LPs. However, any new development in this direction can be immediately incorporated into the absolute gating strategy.

Evaluation of FSC- or SSC-based detection thresholds should also be performed and simultaneously represented in size/RI coordinates. For instance, FSC and SSC channels have similar and remarkably different size sensitivities for RI larger and smaller than 1.53, respectively [Fig. 1(b)]. SSC is probably more sensitive for detecting 200-nm PS beads, but obviously fails to detect MPs with a small RI. While this observation is strictly valid only for the current version of the SFC, it raises some concerns about the validity of any design choices based on reference microspheres (PS or silica), e.g., the conjecture that SSC is more sensitive and suitable for sizing MPs than the FS. ${ }^{15,16}$ In general, any inference from the measurement of one class of particles to that of another class can be considered robust only if made through the realistic optical model of the instrument, as is done in this paper. Note also that the absolute gates in size/RI coordinates are naturally bounded by the FSC and SSC detection thresholds [Figs. 1(b) and 2(a)], which are instrument-dependent. Therefore, to construct a truly standardized interplatform MP gate, one should not try to detect as many MPs as possible, but rather should set the lower-left boundary of the size/RI gate above the detection thresholds of all considered instruments.

Moving to characterization of MPs from scatter signals, we first briefly reflect upon a single-scatter measurements. A widespread belief that FSC is directly related to particle size and SSC is related to granularity is a "gross oversimplification," ${ }^{46}$ which is also evident from Fig. 1. For spherical MPs, rough size estimation can be performed based on Mie theory calculations, if one specifies a fixed RI. ${ }^{18}$ However, the latter is poorly known and can vary inside the sample along with MP composition. ${ }^{47}$ By contrast, simultaneous measurement of both FSC and SSC is known to have a potential for simultaneous determination of size and RI of spherical particles. ${ }^{12,14}$ In this paper, we applied a similar method to submicron particles and, for the first time, directly plotted the number of solutions versus size and RI [Fig. 3(a)]. In particular, for current optical configuration of the SFC, the uniqueness of the inverse Mie problem is provided only for a limited range of MPs, whereas $0.4-\mu \mathrm{m}$ PS microspheres are reliably characterized with no prior assumptions. We also developed an algorithm to estimate the uncertainty of a single solution [Figs. 3(b) and 3(c)], which may be even more important for practical applications then the number of solutions per se. For instance, MPs with RI > 1.42 can be uniquely characterized in a wide range of sizes (up to $600 \mathrm{~nm}$ ) with this particular instrument, but the relative uncertainties are at least 40\%. The examples in Figs. 3(d)-3(g) beautifully illustrate the wealth of the Mie theory behind the dry numbers of Fig. 3(a), but may become a nightmare for practitioners of this method.

Summarizing the above results, we showed that rigorous light-scattering simulations bring the analysis of MPs to a completely new level, in terms of both extracted information and comparability between different instruments. While we cannot directly transfer the specific results (Figs. 1-3) to another instrument, certain problems are expected for any FSC/SSC measuring system, warranting a dedicated analysis. The latter can be performed using the described theoretical framework, completely analogous to the above analysis of the FSC/SSC configuration of the SFC. Moreover, this framework can also be used for optimizing the optical configuration specifically for MP analysis (i.e., to build a dedicated instrument). ${ }^{16}$ This is an important task for future research, but currently it seems daunting as it is both a multiparameter and multigoal optimization. One may wish to have the best sensitivity, ${ }^{45,48,49}$ the smallest characterization uncertainties, the largest range of uniqueness, ${ }^{12}$ or all of these.

Having described a procedure to greatly improve the capabilities of standard FCM in MP analysis, we must admit that this technology has its inherent limits. Part of it is described above with respect to the nonuniqueness of the inverse Mie problem, but can probably be somewhat alleviated by optimization of the optical configuration. By contrast, the major limitation is that two measured numbers can only lead to a maximum of two determined characteristics. Thus, it is not possible to either characterize nonspherical and/or inhomogeneous particles or even to reliably detect the deviation from the simplest homogenoussphere model, which introduces uncontrollable errors in determined characteristics. As shown in Fig. 4, this is especially problematic for polydisperse samples, such as MPs. Unambiguous differentiation of plasma components, such as MPs and LPs, is also very hard since the aggregation may largely distort the determined RI (Fig. 4). Separation of larger MPs from platelets is another problem that cannot be solved by FSC/SSC measurements due to overlapping scatter signals ${ }^{15,42,50}$ in contrast to shape-aware LSP-based analysis. ${ }^{29,30}$

Fortunately, most of the above limitations can be removed by using the next-generation technology (SFC) to measure LSPs of single microparticles. As shown in Figs. 4(c) and 4(f) and previous publications, ${ }^{29,30}$ it allows accurate identification and characterization of MPs by shape, size, and RI with controllable accuracy. The only hardbound limitation is related to the wavelength-dependent Rayleigh limit. All other difficulties are technical ones, related to the complexity of the instrument and corresponding ILS problem. Addressing these technical issues will further enhance the capabilities of the SFC for MP analysis, including better accuracy of RI estimates; this will be reported in another publication.

\section{Conclusion}

We discussed the basic physical principles of scatter-based FCM analysis of MPs and provided a deep insight into actual FCM capabilities and limitations. Understanding the optical model of an instrument and direct simulation of experimental signals is strictly necessary both for adequate data processing using existing instruments and for design (optimization) of future ones. In 
particular, simple gates based on fixed FSC and/or SSC levels cannot reliably identify MPs from a specific size range. We developed a complete theoretical framework for such analysis, but applied it only to a single instrument. Analogous analysis of commercial cytometers, currently used in MPs studies, should be performed to incorporate absolute gating strategy and, hence, to improve the robustness and reproducibility of the results. The presented results also highlight the inherent limitation of standard FCM for MPs, which can be removed only by measurement of additional signals, such as angle-resolved light scattering.

\section{Acknowledgments}

Experimental measurements of platelet-rich plasma were supported by the Russian Science Foundation (Grant No. 14-1500155). Theoretical work, including analysis of the inverse Mie problem, was supported by the Russian Foundation for Basic Research (Grant No. 16-34-00228). D.I. Strokotov also acknowledges the support by the Stipend of the President of Russian Federation for young scientists. The authors declare no conflict of interest.

\section{References}

1. O. Morel et al., "Cellular mechanisms underlying the formation of circulating microparticles," Arterioscler. Thromb. Vasc. Biol. 31(1), 15-26 (2011).

2. N. S. Barteneva et al., "Circulating microparticles: square the circle," BMC Cell Biol. 14(1), 23 (2013).

3. D. Burger et al., "Microparticles: biomarkers and beyond," Clin. Sci. 124(7), 423-441 (2013).

4. S. E. L. Andaloussi et al., "Extracellular vesicles: biology and emerging therapeutic opportunities," Nat. Rev. Drug Discovery 12(5), 347-357 (2013).

5. Y. Yuana, A. Sturk, and R. Nieuwland, "Extracellular vesicles in physiological and pathological conditions," Blood Rev. 27(1), 31-39 (2013).

6. H. M. Shapiro, Practical Flow Cytometry, 4 ed., John Wiley \& Sons, New York (2003).

7. U. Erdbrügger and J. Lannigan, "Analytical challenges of extracellular vesicle detection: a comparison of different techniques," Cytometry A 89(2), 123-134 (2016).

8. J. P. Nolan, "Flow cytometry of extracellular vesicles: potential, pitfalls, and prospects," Curr. Protoc. Cytom. 73, 13.14.1-13.14.16 (2015).

9. P. Harrison, C. Gardiner, and I. L. Sargent, Extracellular Vesicles in Health and Disease, CRC Press, Boca Raton, Florida (2014).

10. P. Poncelet et al., "Tips and tricks for flow cytometry-based analysis and counting of microparticles," Transfus. Apheresis Sci. 53(2), 110-126 (2015).

11. J. Lannigan, J. P. Nolan, and R. M. Zucker, "Measurement of extracellular vesicles and other submicron size particles by flow cytometry," Cytometry A 89(2), 101-220 (2016).

12. D. H. Tycko et al., "Flow-cytometric light scattering measurement of red blood cell volume and hemoglobin concentration," Appl. Opt. 24(9), 1355 (1985).

13. S. G. Ackleson and R. W. Spinrad, "Size and refractive index of individual marine participates: a flow cytometric approach," Appl. Opt. 27(7), 1270-1277 (1988).

14. R. E. Green et al., "Flow cytometric determination of size and complex refractive index for marine particles: comparison with independent and bulk estimates," Appl. Opt. 42(3), 526-541 (2003).

15. F. Mullier et al., "More on: calibration for the measurement of microparticles: needs, interests, and limitations of calibrated polystyrene beads for flow cytometry-based quantification of biological microparticles," J. Thromb. Haemostasis 9(8), 1679-1681 (2011).

16. E. van der Pol et al., "Single vs. swarm detection of microparticles and exosomes by flow cytometry," J. Thromb. Haemostasis 10(5), 919-930 (2012).
17. P. Poncelet et al., "Standardized counting of circulating platelet microparticles using currently available flow cytometers and scatter-based triggering: forward or side scatter?" Cytometry A 89(2), 148-158 (2016).

18. E. van der Pol et al., "Particle size distribution of exosomes and microvesicles determined by transmission electron microscopy, flow cytometry, nanoparticle tracking analysis, and resistive pulse sensing," J. Thromb. Haemostasis 12(7), 1182-1192 (2014).

19. R. A. Dragovic et al., "Sizing and phenotyping of cellular vesicles using nanoparticle tracking analysis," Nanomedicine 7(6), 780-788 (2011).

20. C. Gardiner et al., "Extracellular vesicle sizing and enumeration by nanoparticle tracking analysis," J. Extracell. Vesicles 2(1), 19671 (2013).

21. J. Zwicker, "Impedance-based flow cytometry for the measurement of microparticles," Semin. Thromb. Hemostasis 36(8), 819-823 (2010).

22. F. A. W. Coumans et al., "Reproducible extracellular vesicle size and concentration determination with tunable resistive pulse sensing," J. Extracell. Vesicles 3, 25922 (2014).

23. Y. Yuana et al., "Atomic force microscopy: a novel approach to the detection of nanosized blood microparticles," J. Thromb. Haemostasis 8(2), 315-323 (2010).

24. Y. Yuana et al., "Cryo-electron microscopy of extracellular vesicles in fresh plasma," J. Extracell. Vesicles 2, 21494 (2013).

25. N. Arraud et al., "Extracellular vesicles from blood plasma: determination of their morphology, size, phenotype and concentration," J. Thromb. Haemostasis 12(5), 614-627 (2014).

26. E. van der Pol et al., "Recent developments in the nomenclature, presence, isolation, detection and clinical impact of extracellular vesicles," J. Thromb. Haemostasis 14(1), 48-56 (2016).

27. V. P. Maltsev, "Scanning flow cytometry for individual particle analysis," Rev. Sci. Instrum. 71(1), 243-255 (2000).

28. D. I. Strokotov et al., "Polarized light-scattering profile-advanced characterization of nonspherical particles with scanning flow cytometry," Cytometry A 79(7), 570-579 (2011).

29. A. I. Konokhova et al., "Light-scattering flow cytometry for identification and characterization of blood microparticles," J. Biomed. Opt. 17(5), 057006 (2012).

30. A. I. Konokhova et al., "Super-resolved calibration-free flow cytometric characterization of platelets and cell-derived microparticles in plateletrich plasma," Cytometry A 89(2), 159-168 (2016).

31. V. P. Maltsev, A. V. Chernyshev, and D. I. Strokotov, "Light-scattering flow cytometry: advanced characterization of individual particle morphology," in Flow Cytometry: Principles, Methodology and Applications, pp. 79-103, Nova Science Publishers, New York (2013).

32. J. Fattaccioli et al., "Size and fluorescence measurements of individual droplets by flow cytometry," Soft Matter 5(11), 2232-2238 (2009).

33. C. F. Bohren and D. R. Huffman, Absorption and Scattering of Light by Small Particles, Wiley, Hoboken, New Jersey (1983).

34. M. A. Yurkin and A. G. Hoekstra, "The discrete dipole approximation: an overview and recent developments," J. Quant. Spectrosc. Radiat. Transfer 106(1-3), 558-589 (2007).

35. M. A. Yurkin and A. G. Hoekstra, "The discrete-dipole-approximation code ADDA: capabilities and known limitations," J. Quant. Spectrosc. Radiat. Transfer 112(13), 2234-2247 (2011).

36. S. Xu, J. Liu, and Z. Sun, "Optical factors determined by the T-matrix method in turbidity measurement of absolute coagulation rate constants," J. Colloid Interface Sci. 304(1), 107-114 (2006).

37. D. I. Strokotov et al., "Is there a difference between T- and B-lymphocyte morphology?" J. Biomed. Opt. 14(6), 064036 (2009).

38. A. V. Nichols, "Functions and interrelationships of different classes of plasma lipoproteins," Proc. Natl. Acad. Sci. U. S. A. 64(3), 1128-1137 (1969).

39. D. R. Jones, C. D. Perttunen, and B. E. Stuckman, "Lipschitzian optimization without the Lipschitz constant," J. Optim. Theory Appl. 79(1), 157-181 (1993).

40. A. E. Moskalensky et al., "Accurate measurement of volume and shape of resting and activated blood platelets from light scattering," J. Biomed. Opt. 18(1), 017001 (2013).

41. S. N. Kasarova et al., "Analysis of the dispersion of optical plastic materials," Opt. Mater. 29(11), 1481-1490 (2007).

42. W. L. Chandler, W. Yeung, and J. F. Tait, "A new microparticle size calibration standard for use in measuring smaller microparticles using a new flow cytometer,' J. Thromb. Haemostasis 9(6), 1216-1224 (2011). 
43. C. Gardiner et al., "Measurement of refractive index by nanoparticle tracking analysis reveals heterogeneity in extracellular vesicles," J. Extracell. Vesicles 3, 25361 (2014).

44. G. L. Mills, P. A. Lane, and P. K. Weech, A Guidebook to Lipoprotein Techniques, Elsevier, Amsterdam (2000).

45. R. M. Zucker, J. N. R. Ortenzio, and W. K. Boyes, "Characterization, detection, and counting of metal nanoparticles using flow cytometry," Cytometry A 89(2), 169-183 (2016).

46. G. C. Salzman, "Light scatter: detection and usage," Curr. Protoc. Cytom. 9, 1.13.1-1.13.8 (2001).

47. L. Issman et al., "Cryogenic transmission electron microscopy nanostructural study of shed microparticles," PLoS One 8(12), e83680 (2013).

48. S. A. Stoner et al., "High sensitivity flow cytometry of membrane vesicles," Cytometry A 89(2), 196-206 (2016).

49. T. G. Kormelink et al., "Prerequisites for the analysis and sorting of extracellular vesicle subpopulations by high-resolution flow cytometry," Cytometry A 89(2), 135-147 (2016).

50. S. Robert et al., "More on: calibration for the measurement of microparticles: value of calibrated polystyrene beads for flow cytometrybased sizing of biological microparticles," J. Thromb. Haemostasis 9(8), 1676-1678 (2011).

Anastasiya I. Konokhova graduated from the Physics Department of Novosibirsk State University in 2013. Currently, she is a junior researcher at the Laboratory of Cytometry and Biokinetics, Voevodsky Institute of Chemical Kinetics and Combustion, Novosibirsk, Russia. Her current research interests include flow cytometry and methods for identification and characterization of biological cells and submicron particles in polydisperse systems.

Darya N. Chernova graduated from the Physics Department of Novosibirsk State University in 2016. Currently, she is a PhD student at the Laboratory of Cytometry and Biokinetics, Voevodsky Institute of Chemical Kinetics and Combustion, Novosibirsk, Russia. Her research interests include experimental and theoretical studies of blood microparticles using flow cytometry, including differentiation of various populations of lipoprotein and microparticles.
Dmitry I. Strokotov graduated from the Physics Department of Novosibirsk State University in 2008 . He received his $\mathrm{PhD}$ in biophysics from the Institute of Biophysics, Krasnoyarsk, in 2011. Currently, he is a researcher at the Laboratory of Cytometry and Biokinetics, Institute of Chemical Kinetics and Combustion, Novosibirsk, Russia. His current research interests are focused on scanning flow cytometry and methods for characterization of mononuclear cells.

Andrei A. Karpenko is a head of the Center for Vascular and Hybrid Surgery of Meshalkin State Research Institute of Circulation Pathology. His research interests include endovascular, open, and hybrid operations on major arteries and veins, tissue-engineered constructs and technology in cardiovascular surgery, and cellular and humoral mechanisms of atherosclerosis and thrombosis.

Andrei V. Chernyshev graduated from the Physics Department of Novosibirsk State University in 1988. He received his PhD from the Voevodsky Institute of Chemical Kinetics and Combustion, Novosibirsk, Russia, in 1998. Currently, he is a senior research fellow at the Laboratory of Cytometry and Biokinetics in the Institute. His current research interests are cellular physiology and molecular biokinetics.

Valeri P. Maltsev received his PhD and DrSci degrees in biophysics from the Krasnoyarsk State University and from the Institute of Automation and Electrometry, Novosibirsk, Russia, in 1996 and 2001, respectively. Currently, he is a head of the Biomedical Physics Chair in Novosibirsk State University and Laboratory of Cytometry and Biokinetics at the Voevodsky Institute of Chemical Kinetics and Combustion, Novosibirsk, Russia. His current research is focused on scanning flow cytometry and methods for characterization of biological cells.

Maxim A. Yurkin received his $\mathrm{PhD}$ in computational science from the University of Amsterdam and his PhD in biophysics from ICKC. He is a senior researcher at Voevodsky Institute of Chemical Kinetics and Combustion (ICKC), Novosibirsk, Russia. His current research interests include the discrete dipole approximation, inverse light-scattering problems, and characterization of blood cells. He is the main developer of the ADDA code and received young scientist's award in electromagnetic and light scattering from Elsevier. 